Andrei Rodin

\title{
How Mathematical Concepts Get Their Bodies
}

\section{Introduction}

According to a popular view mathematics progressively becomes more abstract and further detached from intuition. In this paper I shall argue that this view describes adequately only a relatively recent trend which should not be taken as a ground for a long-term strategy. I shall also argue that in spite of this trend the role of intuition in today's mathematics remains by and large the same as it was in Euclid's times. What really changes is not the role of intuition but the mathematical intuition itself. I shall not develop in this paper the philosophical notion of intuition systematically but bring into the fore some mathematical material (both historical and recent) which will make it clearer.

\section{Changing intuition}

Mathematical concepts change rapidly: this change involves both the invention of new concepts and modification of older concepts. Hundred years ago nobody knew what is cohomology (because this concept didn't yet exist) and the notion of algebraic ideal was defined not in the same way as we do it today (see 2.3 below). One can easily learn about such facts studying the old literature. The change of mathematical intuitions is more difficult to notice because intuitions, unlike concepts, rarely allow for precise linguistic descriptions. I shall nevertheless try to show that mathematical intuitions change throughout the history just as dramatically as mathematical concepts.

\subsection{Form and Motion}

It is a truism that in the geometrical Books of his Elements Euclid develops a systematic theory. It is perhaps less obvious that this systematic approach concerns not only geometrical concepts but also geometrical intuitions. The intuitive geometrical content of Euclid's Elements is organised as follows. It comprises (i) primitive intuitions corresponding to concepts of point, straight line and circle and (ii) complex intuitions built with the primitive intuitions. Such combination of primitive intuitions is colloquially called a "construction by ruler and compass". It allows for an intuitive grasp on complex geometrical configurations, which cannot be so grasped immediately. It is not uncommon in the literature to identify the notion of intuition with a primitive immediate intuition but this restriction is not justified. 
Mathematics in general and geometry in particular requires complex intuitions as well as complex concepts.

Let me demonstrate the notion of complex intuition with an example. Think of a regular 1024-gon (polygon with 1024 sides) and proceed as follows. Inscribe a square into a circle. Then by halving angles between diagonals of the square construct an octagon inscribed into the same circle as shown at the below diagram.

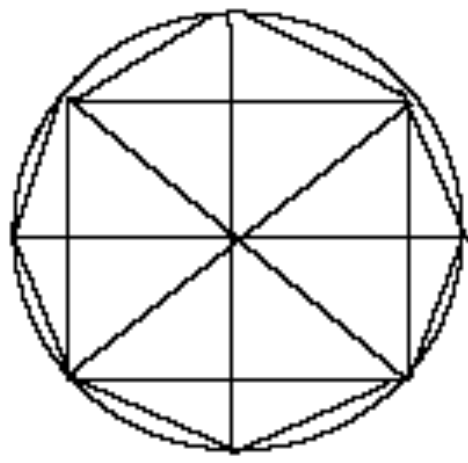

Fig. 1

Then halve the angles between all the neighbouring diagonals of the octagon, get 16-gon and repeat the same procedure 5 more times. (I leave obvious details of this construction to the reader.) Although the resulting figure cannot be grasped immediately it is now well grasped through the above procedure which involves only few primitive geometrical intuitions. Over and above these primitive intuitions it involves an intuition lying behind the very notion of procedure, i.e. of proceeding step by step. This latter intuition is temporal rather than spatial. This construction also requires some basic arithmetical intuitions, which allow one to calculate powers of 2 and thus assure that the number of sides of the obtained polygon is indeed 1024.

A chiligon (1000-gon) is often referred to (after Descartes) as an example of geometrical object, which can be thought of but cannot be imagined. The above example shows that the big number of sides of a polygon does not always make an intuitive grasp impossible. However the method of intuitive grasping used in this example does not work for the chiligon because it cannot be constructed by ruler and compass (Note 1). A similar difficulty concerns a regular heptagon, doubled cube and many other relatively simple geometrical concepts. This 
difficulty shows that the intuitive and the conceptual legs of Euclid's geometry don't always match each other as expected.

The problem was actually twofold. On the one hand, Greek geometers came across a number of geometrical concepts, which they couldn't provide with an intuitive support by standard means, i.e. by performing a construction by ruler and compass. On the other hand, they produced a number of intuitively appealing constructions by other means than ruler and compass but didn't know how to treat them theoretically; I am talking about a cycloid, a spiral and other so-called mechanical curves. (Unlike the circle such curves could not be described without referring to a mechanical setting.) Thus they got a number of problematic cases some of which could be described as poorly intuited concepts while some other could be described as poorly conceptualised intuitions. They applied mechanical curves for solving problems unsolvable by ruler and compass; such solutions pointed to new ways of matching concepts with intuitions. However they didn't develop any alternative systematic theory which could compete with the standard theory based on ruler and compass.

This situation changed significantly only in the Early Modern times. At this point of history the ancient notion of primitive geometrical form lost its earlier appeal, and the idea of construction by ruler and compass was already commonly seen as a pure convention like today. In his Geometry of 1637 Descartes describes an alternative instrument (sometimes called "Cartesian compass") able to produce a large variety of curves other than circles; he argues that constructions made with this new mechanism and the traditional ruler should be treated on equal footing with traditional constructions by ruler and (standard) compass. The Cartesian compass is, of course, only a by-product of the Early Modern reform of mathematics but it well illustrates the fact that mechanical intuitions, which remained in Greek geometry marginal, in the new geometry became central (Note 2).

In the beginning of his Geometrical Lectures of 1735 Barrow claims that all geometrical objects are ultimately generated by motion. Even a brief comparison of Euclid's Elements and Barrow's Lectures reveals that the two works differ not only by their conceptual contents but also by their intuitive contents.

\subsection{Non-Euclidean intuition}

The invention of non-Euclidean geometries in 19th century is often referred as the moment of history when geometry lost its traditional link with empirical spatial intuitions. But this view is wrong. The invention of non-Euclidean geometries in 1830-ies involved the development of new spatial intuitions, not abandoning of spatial intuitions altogether. Moreover this new 
development was conservative: it left the traditional Euclidean intuition untouched. The idea to cut geometry from spatial intuitions came about later, in the very end of 19th century. It soon developed into a powerful trend, which I shall discuss in the next section. The biased view on history of geometry of 19th century mentioned in the beginning of this paragraph is a by-product of this trend.

It is well-known that the discovery of non-Euclidean geometries was a result of long attempts to prove Euclid's 5th Postulate: failing to get a reductio ad absurdum of the negation of this Postulate some people guessed that they were exploring a new vast territory rather than approach the expected dead end. But why did the 5th Postulate attract so much attention to begin with? An obvious answer is that 5th Postulate does not have the same intuitive appeal as other Euclid's first principles; this is why since Antiquity this Postulate was widely seen as problematic. This remark shows that the often repeated claim that the human "natural" spatial intuition is intrinsically Euclidean does not stand against obvious historical evidence. Were this true geometers would always take the 5th Postulate as a self-evident principle along with other Euclid's Postulates and Axioms and wouldn't spent significant efforts trying to prove this particular Postulate on the basis of other principles. It was the mathematical education rather than human nature that later made the majority of people to take the Euclidean intuition for granted, so only the tiny minority of experts could still see and address the problem. In his Geometrical Researches, first published in 1840, Lobachevsky develops the geometrical theory known today after his name combining synthetic constructions à la Euclid with new analytic methods. He produces constructions in a hyperbolic space helping himself with diagrams, on the one hand, and analytic tools, on the other hand; he does not try to mimic hyperbolic constructions by Euclidean constructions or anything else. The reader who knows about Lobachevskian geometry only through its popular models (like Beltrami-Klein and Poincaré models) might be convinced that hyperbolic constructions cannot be drawn by usual diagrams and intuited directly. But in fact they can. The below diagram gives no clue to what happens when the three straight lines shown at the picture are infinitely continued. 


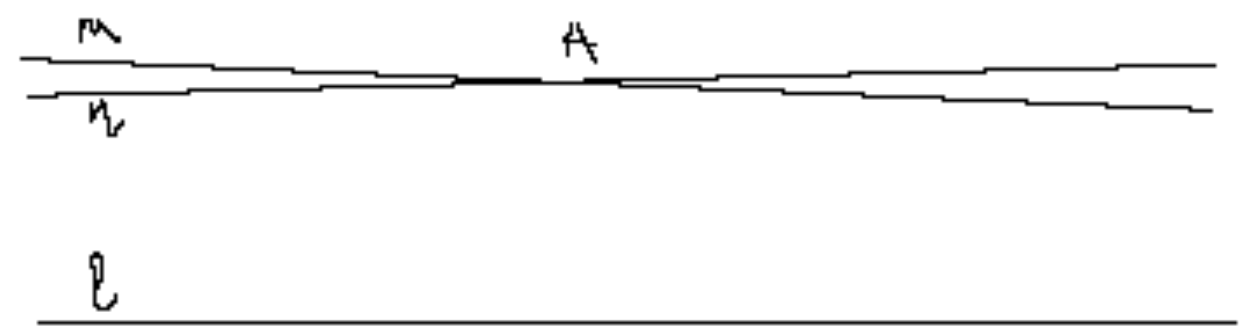

Fig. 2

It may happen and may not happen that at least one of lines $m, n$ will eventually intersect line $l$ at certain point. Assuming that this not happens one may use this diagram as representation of a hyperbolic construction.

In his Saggio of 1868 Beltrami famously identified Lobachevskian plane as a special surface (called pseudosphere) living in the Euclidean 3-space (Note 3). It is less known that earlier in his Researches Lobachevsky used a reciprocal construction: he identified the Euclidean plane as a special surface (horisphere) living in the hyperbolic 3-space. The pseudosphere shows how the hyperbolic plane can be seen through the Euclidean optics while the horisphere shows how the Euclidean plane can be seen through the hyperbolic optics.

The fact that one and the same geometrical object may look differently in different optics motivates the idea of treating geometrical concepts without any appeal to intuition (see the next section). However this is not the only possible and arguably not the best strategy. It is clear that Euclidean and Lobachevskian geometries can co-exist as two logical possibilities. But one may also wish to have a geometrical framework making such co-existence possible. Lobachevsky and Bolyai both looked for such a generalised notion of space which would allow for treating the two cases on common principles.

A satisfactory solution has been proposed by Riemann in his Habilitationsvortrag of 1854; he obtained it through a generalisation of Gauss' theory of curve surfaces. Beltrami identified Lobachevskian spaces as hyperbolic Riemanian spaces (manifolds) very soon after his invention of the pseudosphere (in his Teoria Fondamentale of 1868 - 69). This viewpoint allows one to explain the double nature of the pseudosphere and the horisphere without abandoning geometrical intuitions: each of these surfaces is an embedding of a manifold of one type (Euclidean or hyperbolic) into a manifold of the other type. 
The Riemanian intuition does not reduce to the Euclidean intuition. The best way to get a sense of it is to repeat Gauss' experience which led him to his geometrical discoveries: move around in a hilly environment and try to forget about the third dimension (which is not particularly difficult when the explored territory is sufficiently large). If you need to reach a destination, which is only few feet away, and ask yourself which way is the shortest the answer is obvious: this is the old good Euclidean straight line. But in case the destination is few miles away you need to think hard to find the shortest path and it may turn out that the optimal solution is not unique. We see that the Riemanian geometrical intuition does not cancel the Euclidean intuition but qualifies it as local and extends it with new global features.

Even if the notion of Riemann manifold is dispensable when one needs to map the hilly surroundings of Hanover it is certainly indispensable for mapping the surroundings of our planet at the cosmological scales. So there is not much point in saying that Riemann's generalised notion of geometrical space is "more abstract" than the traditional notion of Euclidean space. People certainly knew about curve spaces long before Riemann and Einstein. What they didn't know was how to treat such spaces mathematically.

\subsection{Lost ideals}

Given a commutative ring $(\mathrm{K},+, \mathrm{x})$ an ideal (in the sense of modern algebra) is a subset I of $\mathrm{K}$ such that I is (1) closed under + and (2) closed under $\mathrm{x}$ in a stronger sense than usual: for all $a$ from $\mathrm{K}$ and all $b$ from $\mathrm{I} a \mathrm{x} b$ is in I. Learning this standard definition a student may wonder where the funny name "ideal" comes from. The definition itself gives no clue to it but historical works provide a clear answer (see Edwards 1980). The term stems from the notion of ideal number (Idealzahl) introduced by Kummer in 1847. Kummer observed that in some rings of cyclotomic integers (Note 4) the unique factorisation into primes fails. His ideal numbers provided the unique prime factorisation in such problematic cases. Kummer called these new numbers ideal because he introduced these objects merely by hand without providing any justification of their existence. This was not something unusual in mathematics: ideal points in geometry, imaginary roots in algebra and even the familiar negative numbers were introduced into mathematics in a similar way. Calling his new numbers ideal Kummer had likely the Kantian notion of ideal in mind (Note 5).

Mathematical ideal elements can be described as bare concepts not supported or only poorly supported by intuition. The history of notion of algebraic ideal shows that this situation can be improved: self-standing "ideal" concepts may acquire appropriate intuitions and so "get 
bodies". In some cases such intuitions can be found among earlier known intuitive contents as it happened when Gauss invented the geometrical interpretation of complex numbers which is nowadays standard. But in some other cases the task requires new intuitions. As we shall now see the case of Kummer's ideal numbers belongs to this second category.

The term "ideal" was introduced by Dedekind; his definition of ideal differs from ours in only one respect: instead of an abstract ring K Dedekind considers cyclotomic rings and other similar rings based on complex numbers. Dedekind showed that Kummer's ideal numbers can be identified with his ideals of a special sort, i.e. as certain infinite classes of complex numbers (hence the name).

Dedekind's notion of ideal in comparison with our modern notion of ideal strikes as too special and even "concrete". One should not however forget that considering infinite collections of mathematical objects (like complex numbers) as mathematical objects in their own rights is a relatively recent idea. In Dedekind's times it was very new and even revolutionary. It came about with the emerging Set theory. Dedekind's and our modern definitions of ideal no longer seem to be relevant to the philosophical notion of ideality because in 20th century people learnt to operate with infinite collections in a manner similar to which they earlier operated with triangles, natural numbers and complex numbers (which also at certain point of history lost their "imaginary" flavour). When I say that people operate with infinite sets like with numbers and triangles I mean that in all these cases corresponding concepts (set, number, triangle) are supported by intuitions which make these concepts into full-fledged mathematical objects. When one adds two numbers or inscribes a circle into a given triangle one does not operate with bare concepts; the involvement of intuition in such cases is hardly disputable. When one takes a powerset of a given set - no matter finite or infinite - the pattern is the same: one works with the given set but not with the concept of set.

\section{Flight from intuition and Set theory}

The idea that intuition is a shaky matter, which must be avoided in science and mathematics as much as possible, is very ancient. But in the end of 19th this old idea took a very particular twist, which turned to be very important for mathematics of the following century. In 1899 Hilbert published his Foundations of Geometry on which he commented as follows:

"[I]t is self-evident that every theory is merely a framework or schema of concepts together with their necessary relations to one another, and that basic elements can be construed as one pleases. If I think of my points as some system or other of things, e.g. the system of love, of 
law, or of chimney sweeps $<\ldots>$ and then conceive of all my axioms as relations between these things, then my theorems, e.g. the Pythagorean one, will hold of these things as well." (quoted by Frege 1971)

This quote supports the following view: geometry ultimately does not depend on the intuitive images usually associated with terms "straight line" and "point", so one may imagine these things in any way one likes or avoid imagining them altogether; even if intuition is quite useful for doing mathematical research it must play no role in justification of results of this research. Let me now show that in fact intuition continues to play its fundamental role even if a theory fully meets Hilbert's standard just described.

In the very beginning of his Foundations Hilbert describes the domain of his new geometry as a family of "systems of things". The notion of system of things seems very abstract and detached from any intuition. And it indeed has this character as far as it remains a subjectmatter of logic and metaphysics. But when it becomes a part of mathematics the situation immediately changes. I shall demonstrate this by considering two different mathematical ways of thinking about a system of things: as a set and as a category. I shall discuss sets in this section and categories in the next section.

Cantor invented Set theory trying to make up a notion of infinite or "transfinite" number, which could help one to account for infinite sets of points on a given line. This pioneering work involved not only invention of new concepts but also development of new intuitions. Consider for example how Cantor introduces infinite ordinals in his Grundlagen einer allgemeinen Mannifaligkeitslehre of 1883. He gives the name of first principle of generation to the usual operation $(+1)$ which allows one to get any finite number $n$ starting with zero. Cantor takes this operation for granted, i.e. he understands it in the usual intuitive sense. Then he introduces his second principle of generation, which amounts to stipulation of an infinite limit $w$ of the natural series of all finite ordinals. Then he again applies the first principle and obtains numbers of the form $w+1, w+2$, etc. The combination of the two principles brings about $2 w, 2 w+1, \ldots 3 w, . ., w^{2}$, etc. Clearly Cantor purports here to extend the traditional intuition about counting with a new intuition of "infinite counting". He explicitly appeals to the reader's imagination in this paper.

One may agree that Cantor's work involved a good deal of imagination but argue that this intuitive aspect of Set theory lost its significance when Set theory was put on the strict axiomatic basis by Zermelo and others. However this argument does not go through. The axiomatic treatment does not help one to get rid of set-theoretic intuitions in the same way in 
which it may help to get rid of traditional geometrical intuitions. (The relevant notion of axiomatic method is one described in the above quote from Hilbert.) For this method, as we have seen, requires some notion of "system of things" to begin with. Sets in the precise sense of ZF or any other axiomatic theory of sets are systems of "well-behaved" things, which don't lead to known paradoxes. The distinction between sets and infinite collections of a more general sort is, of course, essential but it does not help avoiding intuitive thinking about sets. It may only guide and refine our pre-theoretical intuitions about sets.

The intuitive ground of axiomatic theories of sets can be described more specifically through an analysis of particular set-theoretic axioms. Consider, for example, the axiom of pairing making part of ZF. This axiom says (in words) that given sets $x, y$ there also exists set $\{x, y\}$ having the given sets $x, y$ as its elements. This axiom has been little discussed in the literature because it is generally seen as unproblematic. But what makes it so unproblematic? What justifies it as a principle?

There is at least two different sorts of justification one may think of in this case. Some people (Fregeans) would take seriously the question whether or not the pairing axiom is true. Other people (Hilbertians) would argue that in the modern axiomatic setting the question whether or not some particular axiom is true makes no sense; they would argue that any system of axioms is good if it is logically consistent. But however significant the difference between these two approach might be it does not really matter in the given case. Since the consistency of ZF cannot be proved without taking a stronger theory for granted there remain only this way to save this theory: one assumes the consistency of ZF as a working hypothesis and waits until an eventual contradiction shows up; if this will happen in the future people will think how to modify the ZF in order to avoid this particular type of contradiction. (This is how ZF took its present shape: the axioms of this theory prevent known paradoxes but offer no remedy against possible unknown paradoxes.) But this pragmatic decision requires some additional grounds, which could make the hypothesis of consistency of ZF at least plausible. There is no other way to estimate the plausibility of this hypothesis except estimating the plausibility of axioms of ZF and of known consequences of these axioms. The Fregean party uses exactly the same source for defending its cause. Both parties see pairing as unproblematic. The fact that the two parties understand "unproblematic" in slightly different ways does not effect my argument.

I claim that pairing appears unproblematic because it appeals to a common practice of manipulation with middle-size objects. It is easy to reproduce such manipulations with symbols: take $x$, take $y$, use \{\} , and combine these symbols into $\{x, y\}$. Thus pairing has the 
same kind of intuitive appeal as Euclid's first Postulate: it is something one can easily do or imagine doing.

I shall not consider other set-theoretic axioms here but point to few general difficulties. First, the apparent intuitive appeal of the notion of set often turns to be misleading even in finitary cases. In particular, the difference between set $x$ and the singleton $\{x\}$ does not correspond to any intuitive difference; particularly mysterious from an intuitive viewpoint is singleton $\{0\}$. Second, there is a clash between the generality of the set concept and the specific character of intuition usually associated with this general concept. Even if thinking of geometrical objects as sets of their points is not plainly contradictory (as people believed before Cantor) such thinking may be inappropriate for different reasons. Arguably it imposes a specific pointbased intuition and rules out other intuitions which could be more useful for supporting geometrical concepts associated with these objects.

These remarks show that the intuitive view on Set theory in its existing form can be hardly developed systematically. This is not particularly surprising since the intuitive aspect of this theory was not taken seriously by creators of axiomatic theories of sets and of set-theoretic foundations of mathematics. The common idea was not to avoid using intuitions in mathematical practice (which is not realistic) but rather treat this use of intuition as an art or a skill rather than a part of theory. Thus the set-theoretic foundations brought about some specific and often confusing intuitions, which I have tried to describe above, but provided no systematic guide for using them.

\section{Towards categorical foundations}

Category theory appeared on the scene in 1950-ies as a very general "language" useful in various domains of mathematics. In early 1960-ies Lawvere put forward a continuing project of building new foundations of mathematics with Category theory described in his paper of 1966. I shall not consider here the controversy between set-theoretic and category-theoretic foundations of mathematics systematically but stress the role of intuition in Category theory and argue that the ongoing project of building category-theoretic foundations has no reason to follow the strategy of flight from intuition.

The notion of category (in the mathematical sense of the term) can be seen as another mathematical realisation of Hilbert's "system of things". Looking at "things" which came into the mainstream mathematical research of 20th century one notices the following: they have an associated notion of map or structure-preserving transformation. We have already seen this in 2.2 at the example of Riemanian manifolds. Think of sets and functions (maps between 
sets), topological spaces and continuous transformations, groups and group homomorphisms, vector spaces and linear transformations. The notion of category describes this general situation: a category comprises abstract objects, maps between these objects (also called morphisms) and a notion of composition of maps, which is a subject to few axioms. Having only this notion of category in hands one can immediately think of further categorical constructions by considering given categories as objects of some other categories. (One needs for it some notion of morphism between categories; such morphisms are called functors). Let us consider more precisely the categorical notion of composition and its intuitive content. This intuitive content which combines spatial (geometrical) and temporal (algebraic) intuitions can be made clear with the following diagram.
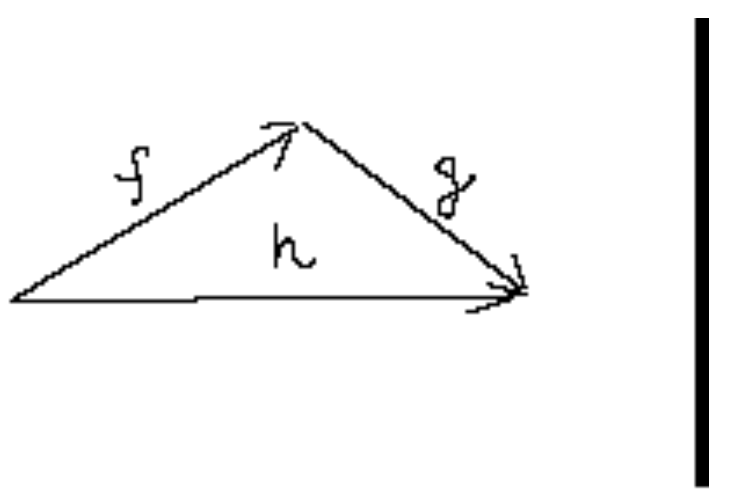

Fig. 3

Vertexes of this triangle stand for objects while its edges stand for morphisms between object. The lower edge stands for the composition of the two morphisms represented by the two upper edges. The morphisms are composed in the "geometrical" order, which becomes obvious when one "follows arrows". This composition can be also written in symbols as $f^{*} g$ (or $g^{*} f$ in a more popular notation). However this simple algebraic notation hides the fact that in the given case the operation * is, generally speaking, only partial. This means that two given morphisms cannot be always composed even if they belong to the same category: the composition $f^{*} g$ exists if and only if the target object $B$ of $f$ (i.e. codomain of $f$ ) coincides with the source object $B$ (i.e. domain) of $g$. From a geometrical point of view this feature of categorical composition can be also described as local ; a precise topological sense of "local" can be provided through the notion of Grothendieck topology which defines neighbourhoods of objects of a given category in terms of their incoming morphisms. The idea of "localisation" of classical algebraic structures can be compared with the localisation of Euclidean geometry within Riemanian geometry discussed in 2.2 above; it is fundamental for 
Sheaf theory, which made a new start in 1960-ies when Grothendieck and others began to use in it categorical methods. I mention here these developments (even if I cannot discuss them here more precisely) for stressing the following: the match of geometrical and algebraic intuitions in the notion of category implies much more than a cognitive comfort; it supports a new ongoing conceptual synthesis of geometric and algebraic legs of today's mathematics. I shall explain the idea of categorical foundations by an analogy with the Hilbert-style axiomatic method, which hereafter I shall call "standard".

Remind that the standard method amounts to stipulation of certain formal properties of (abstract) relations between (abstract) individuals. For example, axioms of ZF involve only one such (non-logical) relation, namely that of membership. Any elements of some abstract set $M$ and any relation $R$ between these elements which has the formal properties required by axioms of ZF (for example the relation of membership in $M$ ) can be called sets in the precise sense of ZF provided that $R$ is interpreted as their relation of membership. (The reader may notice that this standard construction involves a suspicious circularity but I shall leave this problem aside.) An axiomatic theory built with Category theory assumes in its turn that its objects are objects of some (abstract) category and then stipulates some general properties of this category. Taking sets to be "black boxes" linked by abstract morphisms and stipulating a number of properties of these morphisms one gets a category, which can be identified as the category of sets (Note 6). We see that in the categorical method morphisms play a role similar to that played by relations in the standard axiomatic method. When one understand the notion of relation in a wide philosophical sense it seems indeed appropriate to say that morphisms "relate" objects to each other. However morphisms are not relations in the usual sense of modern logic: while usual relations are defined "globally" for a given set of relata each morphism is determined "locally" with respect to its source and target. From the standard point of view of modern logic categorical morphisms are also "objects" of a sort rather than relations. This technical detail shows that in spite of the obvious similarity none of the two methods immediately reduces to the other (although each of them can be mimicked by the other).

Let me now explain why I think that the categorical method (which admittedly does not exist to the present date in an accomplished form) does not need to "fly from intuition" after the standard method. Remind that the standard method involves the notion of set as an abstract replacement for traditional mathematical objects. When Set theory was made into foundations of mathematics this abstract character of the notion of set was at least partly forgotten and working mathematicians began to think of sets as something concrete. The notion of category 
repeats the same pattern. It first strikes as excessively abstract and general; this is the reason why it was infamously called "abstract nonsense". This attitude is understandable when one thinks of categories through "concrete examples" like the category of sets, of topological spaces, etc. Even one who can take the powerset of a given abstract set just as easily as produce a straight line from any given point to any given point can be confused by the proposal, first, to think of all sets and functions, then forget how any particular set looks like, and finally make further constructions with the obtained highly abstract notion. Dedekind contemporaries had a similar impression of his set-theoretic definitions of ring and ideal, which today look unproblematic and even too "concrete". However when one makes Category theory into foundations this abstract character of the notion of category disappears just like in the case of sets. Then instead of thinking of a categorical morphism as a very abstract notion covering functions, continuous transformations and the like one conceives of these morphisms as elementary building blocks. In this case morphisms can be immediately intuited in the way which I have already explained. This does not mean, of course, that the mathematical content related to these older "concrete" examples can and should be forgotten but this means that this content should be rebuilt on the new foundations. I have already explained generalities making such reconstruction possible. I would like to add that new foundations allowing only for the reconstruction of older notions are of little interest unless they allow for solving problems, which couldn't be solved by older methods. In this latter sense the case of categorical foundation still remains disputable but each year brings new mathematical results making more people to judge in its favour (Note 7).

The qualification of mathematical objects as abstract entities goes back to Aristotle. On Aristotle's account any mathematical object is abstract in the sense that it represents some specific feature or combination of features of physical objects. But in the modern mathematics sets and set-based structures are called abstract in a much more specific sense, namely in the sense that such structures have multiple interpretations and allow for "concrete examples" within mathematics. This very specific notion of abstraction and the related notion of mathematical structure allowed for a very productive cross-fertilisation between different fields of mathematics; it also allowed for an impressive unification mathematics (see Bourbaki's Architecture of 1950). This obvious success provides a strong argument in favour of the Hilbertian strategy of flight from intuition independent of any further philosophical reason. But Category theory achieves a similar effect by different means without appealing to higher abstractions. A model or interpretation of category $A$ in category $B$ is a functor of the form $A-->B$. This notion of interpretation (which can and should be further specified) allows 
for treating categories $A$ and $B$ on equal footing without considering one of them as abstract and the other as concrete. While in the standard set-theoretic setting the notion of interpretation is treated by the separate meta-mathematical discipline of model theory and is often left without any precise treatment in the rest of mathematics in the categorical setting it becomes a typical construction treated on equal footing with constructions of different types. Thus categorical foundations allow for avoiding the artificial division of mathematics into the meta-matematics and the mathematics proper (Note 8).

\section{Conclusion}

Talking about mathematical intuition in this paper I deliberately left this central notion without any precise definition. On the one hand, I certainly tried to avoid the popular understanding of intuition as a bunch of vague ideas and motivations accompanying any intellectual advance. But on the other hand, I also didn't want to use in my discussion any philosophically refined notion of intuition like one developed in the phenomenological tradition. This would make it difficult to describe the role of intuition in mathematics remaining sensitive to differences between different types of mathematical theories and different foundations of mathematics. Perhaps one can formulate a more general notion of intuition, which remains stable through all the historical changes described in this paper. I shall not purport here this project but only stress that matching concepts with intuitions always was and, in my view, will always remain a principle purpose of mathematics. In his Critique of Pure Reason Kant famously observes that "thoughts without content are empty, intuitions without concepts are blind" (B75) and then shows how concepts and intuitions work together in mathematical thinking. But such a match between concepts and intuitions is not given us for free in our mental constitution as a Kant's reader might wrongly assume; it is repeatedly reached more or less successfully in mathematical practice. This match makes possible the specific kind of clarity and precision called "mathematical clarity" and "mathematical precision", which is both impossible and inappropriate in the domain of a purely speculative philosophical thought. Barring intuition from foundations of mathematics inevitably results into the lost of this specific mathematical clarity and precision, which is regrettable not only for mathematics itself but also for mathematically-laden science. As I have tried to show in this paper the situation is not hopeless: even today's "abstract" mathematical concepts can be matched with appropriate intuitions. In order to make a progress in this direction one should, first of all, recognise the fundamental epistemic role of intuition in mathematics rather than treat intuition as a merely pedagogical and heuristic issue. 
The pure speculation in its turn has its own criteria of clarity and precision, which are not the same as in mathematics. Although philosophical considerations can be eventually useful in mathematics and mathematical consideration can be eventually useful in philosophy it seems me important always to keep in mind that philosophical speculations cannot count as mathematical proofs while mathematical proofs cannot count as philosophical arguments.

\section{Endnotes:}

1) As it has been first conjectured by Gauss and proved in 1837 by Wintzel a regular polygon is constructible by ruler and compass if and only if its number of sides is a product of any power of 2 and a Fermat prime. Fermat primes are primes of the form $(2 \exp 2 \exp n)+1$ where $n$ is a positive integer.

2) Descartes does not give up the ancient distinction between mechanical and (properly) geometrical curves altogether but changes its sense: on Descartes' account only non-algebraic curves qualify as mechanical. Following the ancient pattern Descartes claims that such mechanical curves do not belong to the subject-matter of pure geometry. However this ban of transcendental curves was hardly ever respected in Modern mathematics.

3)This was, strictly speaking, a mistake: the pseudo-sphere is not a model but only a partial model of Lobachevskian plane.

4) A ring of cyclotomic integers is the ring of usual integers extended with $p$ th root of unity where $p$ is a prime number.

5) I leave aside the question whether or not mathematical ideal elements indeed qualify as ideal in Kant's sense. It does not have any obvious answer because in Kant's understanding mathematics does not allow for ideal elements.

6) This result was first published by Lawvere in his paper of 1964. It obviously calls for further qualifications which I skip here.

7) Among recent examples I shall point to works of Khovanov (see his paper of 2000) who improved on the classification of knots using the method of categorification.

8) The Hilbert-style axiomatic method is an essential element of structuralist approach to mathematics. My description of the categorical method as a genuine alternative to this standard method may provide some justifications for my belief that the category-theoretic mathematics is not structuralist as it is often claimed. However I cannot develop this point in this paper. 
Bibliography:

Beltrami, E., 1868, "Saggio di interpetrazione della geometria non-euclidea", Giornale di Matematiche, v.6, p. 284-312

Beltrami, E., 1868-69, "Teoria fondamentale degli spazii di curvatura constante", Annali di matematica pura et applicata (2), 2, p. 232-255

Bourbaki, N., "The Architecture of Mathematics" The American Mathematical Monthly, Vol. 57, No. 4. (Apr., 1950), pp. 221-232.

Cantor, G., 1883, Grundlagen einer allgemeinen Mannifaligkeitslehre: Ein mathematischphilosophischer Versucht in der Lehre des Unendlichen. Leipzig, Teubner

Edwards , H. M., 1980 "Genesis of ideal theory", Arch. Hist. Exact Sci., 23, pp. 321-378

Frege, G., 1971, On the Foundations of Geometry and Formal Theories of Arithmetic, E.H.W. Kluge (ed.), Yale University Press

Khovanov, M., 2000, "A categorification of the Jones polynomial", Duke Mathematical Journal 101 (2000) 359-426. (arCiv:math.QA/9908171.)

Lawvere, W., 1964, "Elementary Theory of the Category of Sets", Proceedings of the National Academy of Science, vol. 52, N6, p.1506-1511

Lawvere, W., 1966, "The Category of Categories as a Foundation for Mathematics", La Jolla Conference on Categorical Algebra, Springer-Verlag, p.1-20

Lobachevsky, N.I., 2007, Geometrical Researches on the theory of Paralles (transl. Halsted)

Riemann, B., 1854, "Ueber die Hypothesen, welche der Geometrie zu Grunde liegen” (Habilitationsvortrag), Abhandlungen der Kgl. Gesellschaft der Wissenschaften zu Göttingen. 13 (1867): 133-152; reprinted in Gesammelte Mathematische Werke (Narasimhan, ed.), Berlin, Heidelberg, NewYork: Springer-Verlag, 1990 